\title{
Quantitative morphological studies of developing human cerebellar cortex in various disease states
}

\author{
DEREK R. GADSDON and JOHN L. EMERY \\ From the Department of Histopathology, the Children's Hospital, Sheffield
}

\begin{abstract}
Gadsdon, D. R., and Emery, J. L. (1976). Archives of Disease in Childhood, 51, 964. Quantitative morphological studies of developing human cerebellar cortex in various disease states. A quantitative morphological assessment was carried out of the cellularity and staining properties of the cells of the layers of the human cerebellar cortex, both in the normal child and in 41 children suffering from a series of disorders including mental retardation. A computerized image analyser and highly standardized procedures were used.

All of the cases of mental retardation and some cases with congenital cardiac anomalies showed abnormal cell concentrations and staining properties. 3 cases of 'cot death' also showed abnormal results. These findings are presented as a new measurable aspect of brain disease, and as an indication for further study.
\end{abstract}

There has been much to indicate that the cellularity of the developing cerebellum can be altered by a variety of agencies; hypothyroidism (Balázs, 1972), cycasin (Hirono, Shibuya, and Hayashi, 1969), methylazoxymethanol (Shimada and Langman, 1970), thiofuran (Herndon, 1968), alkyl mercurial halides (Hunter and Russell, 1954), diphenylhydantoin (Kokenge, Kutt, and McDowell, 1965), heat stroke (Krainer, 1949), and mild undernutrition (Dobbing, Hopewell, and Lynch, 1971). The evidence in man, however, is largely based on subjective impressions of altered cell populations, or on nonmorphological studies.

The recent development of a television-based image analysing computer, capable of the detailed analysis of histological preparations, has enabled us to make more accurate quantitative assessments of the concentration, size, and staining properties of brain cells. Using this instrument, a Quantimet 720 (Cole and Bond, 1971), we have determined the postnatal changes in the cellularity of the external granular, internal granular, and molecular layers in the human cerebellum in 'normal' children (Gadsdon and Emery, 1976a) and were able to show that specific lobules of the cerebellum are damaged in children suffering from hydrocephalus with spina bifida (Emery and Gadsdon, 1975). In establishing the normal range of values it was necessary to exclude any child with known mental deficiency.

Received 7 March 1976.
We present here findings in children with known mental retardation, together with comments on other children producing values below the 5th centile of the normal series.

\section{Material and methods}

The method of preparation of material and of carrying out the counts has already been described (Gadsdon, 1976). Ten 12- $\mu \mathrm{m}$ sections from a standardized block of the lateral lobe were prepared from each of the 489 cerebella studied, and critically stained with a controlled Feulgen technique for DNA. Cell counts were carried out using a television-based image analysing computer (Quantimet 720) which was programmed to calculate the number and size of the nuclei in a unit area in the different layers of the cerebellar cortex. It must be stressed that the results are based upon the number of cells per unit area, not volume. Thus the terms 'concentration' and 'cellularity' referred to in the text describe the number of cells per unit area.

The areas studied were the external granular, the molecular, and the internal granular layer. In every cerebellum studied 200 readings were taken in each of the lay ars and the results were expressed as the number of cells $/ \mathrm{mm}^{2}$, except for the external granular layer. In this layer, as it eventually becomes a single cell layer and finally disappears, counts were therefore expressed as the number of cells/mm length of cerebellar surface. With the exception of the exclusion of the Purkinje cells, no attempt was made to differentiate between any cell type.

The optical density of the Feulgen-stained nuclei of the internal granular layer was also estimated using the 
same cells as those used for the cell counts, and expressed as the average optical density per unit area of the nuclei from that particular cerebellum. This equates ideally to the amount of DNA in those nuclei.

When the measurements were made the operator was completely unaware of the age of the child, the clinical history, or any pathological data.

In our assessment of the normal concentration of cells in the cerebellum in relation to age, normality was assumed when there was no clinical history of neurological disorder, no overt histological evidence of significant neuropathology, and where the weight of the cerebellum was within normal limits for the age. Thus, for the purpose of that study all cerebella showing congenital deformities were excluded. The normal ranges were derived from the rest of the cerebella. This of course required exclusion of a large number of children with hydrocephalus and meningomyelocele, which formed the basis of an earlier study (Emery and Gadsdon, 1975) where it was noted that the most consistent parameter of abnormality was the reduced cellularity of the internal granular layer. The cell counts obtained from the internal granular layer were therefore used in the selection of cases for this study.

The present report concerns a total of 41 children. 24 of the children presented with clinical evidence of mental retardation, yet histologically overtly normal brains. The causes of death of these children included pneumonia, gastroenteritis, septicaemia, and renal failure. None of these cases showed evidence of any congenital cardiac anomaly. The other 17 children included in this report presented with no significant neurological history and histologically normal brains, but quantitative studies of the internal granular layer showed cell concentrations below the 5th centile of our 'normal' population. Of these children, 12 had severe congenital deformities of the heart. One other child had multiple congenital anomalies including a tracheo-oesophageal fistula, associated with repeated periods of respiratory distress. There were 3 cases of unexpected death in infancy, all of which fell into the classification of group B (Emery, Swift, and Worthy, 1974), i.e. dying from recognizable and apparently treatable disease, but none of these cases showed overt evidence of brain damage. The final case was a child who died from neonatal herpes in which there were extensive areas of necrosis in the liver, but naked eye appearance and routine histology of the brain showed no evidence of encephalitis.

The weights of the cerebella and the cerebellum/brain weight ratios were within normal limits for 22 of the 41 children studied. The remaining 19 cases showed slightly reduced cerebella weights and ratios, and all were from children with mental retardation.

\section{Results}

The cell concentrations in the internal granular layer of the 41 children reported here are shown in Fig. 1 where their cell counts are related to the normal developmental pattern for this layer (Gads-

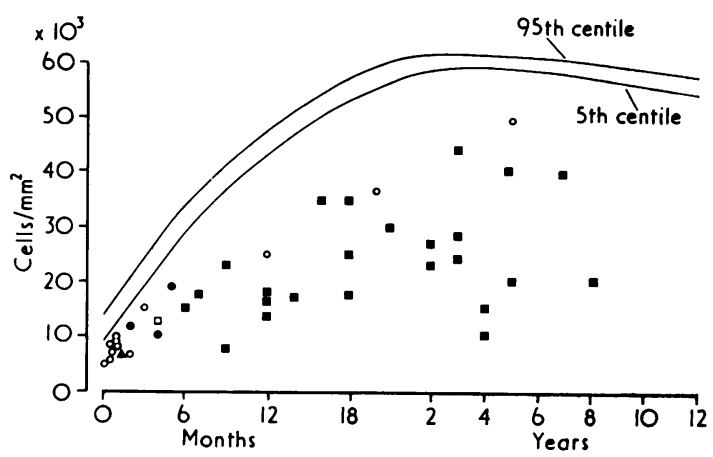

FrG. 1.-Cell concentration $/ \mathrm{mm}^{2}$ in the internal granular layer of the cerebellum. The 95th and 5th centiles were obtained from 354 'normal' children. ( $\square$ ) mentally retarded children; (O) congenital cardiac anomalies; (O) children dying as 'cot deaths'; ( $\square$ ) a case of herpes simplex; (A) a child with multiple congenital anomalies. The age scale in this and all subsequent figures is expanded for the first 2 years of life.

don and Emery, 1976a). All of the cases show reduced cell concentrations. Fig. 2 shows the cell concentrations of the molecular layer, where the 41 cases are recorded against the 5th and 95th centile curves. The majority of the 41 cases fall within the normal range; however, 11 of the 24 mentally retarded cases show abnormal cell concentrations. When the 41 cases are identified on the graph of the external granular layer (Fig. 3), the majority of the cases show an abnormal cell concentration, $75 \%$ of the mentally retarded cases having altered cell counts.

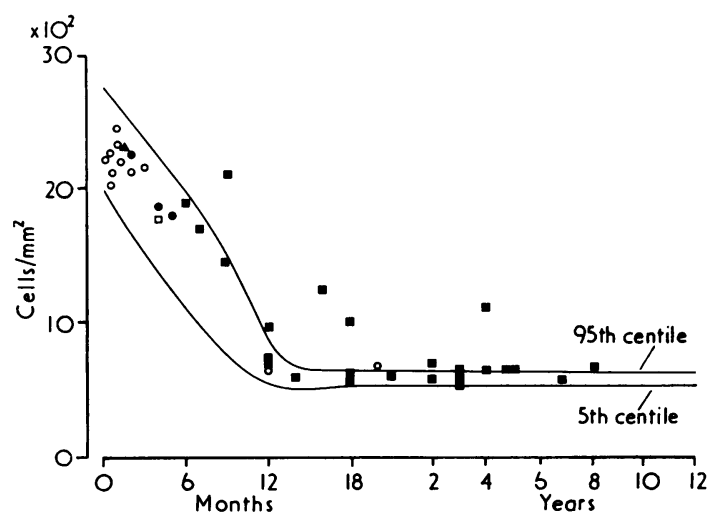

FIG. 2.-Cell concentration $/ \mathrm{mm}^{2}$ in the molecular layer of the cerebellum. See Fig. 1 for explanation of symbols. 


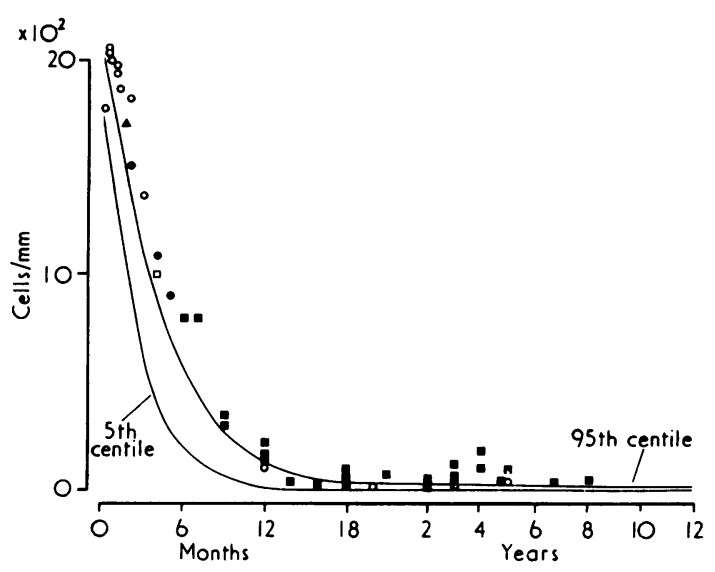

FIG. 3.-Cell concentration/surface $\mathrm{mm}$ of cortex in the external granular layer of the cerebellum. See Fig. 1 for explanation of symbols.

The 41 cases are also identified on the centile curves for the absolute optical density values of the Feulgen-stained nuclei of the internal granular layer (Fig. 4). These values ideally equate to the concentration of DNA within the nuclei of the cells examined. 38 of the 41 cases show reduced optical density levels; all of the 24 mentally retarded cases show reduced levels, including all of the most markedly abnormal values.

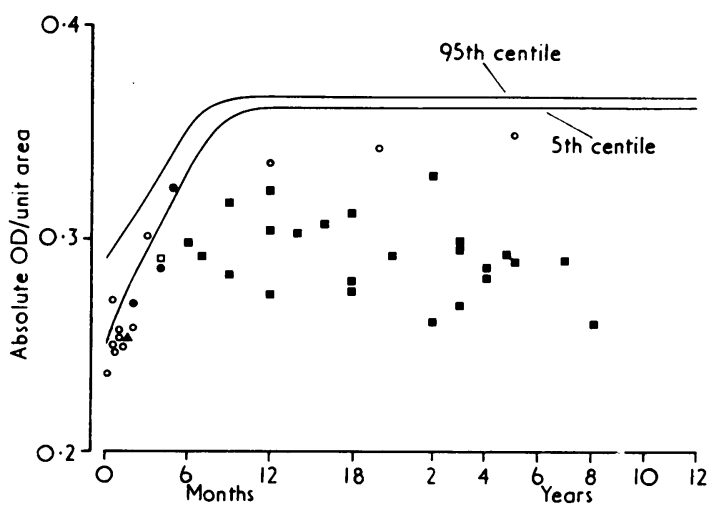

FIG. 4.-Absolute optical density (OD) per unit area of the Feulgen-stained nuclei (DNA) of the internal granular layer of the cerebellum. See Fig. 1 for explanation of symbols.

\section{Discussion}

The results must be related to the development of the three layers of the cerebellar cortex. Maturation of the cortex involves the migration of cells from the external granular layer through the molecular layer into the internal granular layer.
As this migration appears to be largely complete by the age of 2 years, the most important criterion is that of the final state of the internal granular layer. The number of cells in sections of the molecular layer is a measure of the number of cells in migration at that particular time, and this can be affected by at least two factors: the number of cells migrating and the rate at which migration is occurring. Thus the actual number of cells seen in the molecular layer may bear no direct relation to the number of cells eventually available to the mature internal granular layer. The number of cells in the external granular layer at the time of birth and probably a little before that represent the potential number of cells awaiting migration to the internal granular layer and thus the potential number available for maturation. An alteration from the normal number of cells present in the external granular layer is theoretically affected by two factors. (1) The number of cells primarily present, and (2) the rate at which cells have left this layer; thus a delayed migration of cells could result in an apparent increase in the number of cells per unit area. At the moment we have no means of knowing which factor is determining the cell concentration in this zone.

Thus, our final interpretations need, at the moment, to be based upon changes in the internal granular layer. Here we see a striking picture. All of the children with recognized clinical mental deficiency showed reduced cell concentrations and apparently lowered concentrations of DNA within the cells of the internal granular layer. These children showed no specific clinical or histological cerebellar defects, though 19 of these children had slightly reduced cerebella weights. We have not as yet carried out quantitative morphological studies of other parts of these children's brains, but it seems most likely that we are studying a nonspecific cell depletion in the cerebellum.

The finding that in 17 children with depleted cell concentrations, 12 had congenital cardiac anomalies, requires further study. The brains of these children were superficially normal, and the small areas of calcification found in the corona radiata had not been considered to be of sufficient rarity to consider the brains abnormal. The series of children whose brains we used in our 'normal' studies (Gadsdon and Emery, 1976a) contained some with congenital deformities of the heart. We had excluded from the normal series several children with cephalic cysts and simple hydrocephalus associated with congenital deformities of the heart, as brain degeneration in children with such deformities is well recognized clinically and patho- 
logically. The implication now is that all children with congenital deformities of the heart should not be assumed to have 'normal' brains even if the brain has been superficially described as normal. Further studies of these brains are urgently required, in view of the present tendency to operate on an increasing number of these children, assuming that their defects are confined to the heart.

The reduced cell concentrations and DNA concentrations per unit area of nucleus observed in the 3 cases of unexpected death in infancy (type B) cannot be explained. The 3 children had apparently normal brains, though all showed some fatty change in the cerebral hemispheres, indicating a possible derangement of myelinogenesis involved in hypoxia (Gadsdon and Emery, 1976b).

Following our finding that the cerebellar cortex of the child with a herpes infection showed reduced cell concentrations, the brain sections were resurveyed. A small number of cells with intranuclear inclusion bodies characteristic of herpes was found, and this child was thus both subclinically and, from a routine histology viewpoint, subhistologically a herpes encephalitis. The infection in this case undoubtedly occurred before birth.

The final abnormal case was that of the child suffering from multiple congenital anomalies. Though the child had no neurological history and an apparently normal brain histologically, the results we present may be due to a subtle congenital anomaly of the cerebellum, or to changes associated with respiratory distress.

In our studies of the cerebella from children with meningomyelocele (Emery and Gadsdon, 1975) a notable feature was an apparent reduction in the concentration of DNA per unit area of nucleus of the nuclei of the cells of the internal granular layer. Reduced levels of DNA compared with the normal range are a feature of 38 of the 41 cases in the present study, using the DNA methods employed in this investigation. A detailed investigation into the exact nature of the DNA results presented here and in our other papers is at present in hand, and it must be emphasized that the apparent DNA changes we have reported in these studies could be due to several causes, including changes in nucleoprotein composition and the volume of the nucleus. However, it seems that whatever the exact reason for these alterations in Feulgen-staining, we have a useful sensitive parameter of nuclear abnormality.

We now apparently have a histological method for measuring subclinical brain damage, though at the moment we have no means of determining any specificity in this direction, or evidence as regards aetiology. The importance of the results presented here indicates the possibility of making quantitative estimations of the brain at necropsy and relating this to the many facets of potential developmental interference, including mental retardation.

At the present state of development of computerized image analysis equipment and its associated histotechnology, attempts to differentiate automatically between the cell types of the cerebellar cortex are largely limited, and a great deal of further work must be carried out. However, it was obvious that the greatest proportion of cells involved in the cellular alterations of the internal granular layer in the 41 cases described above was the granule cell. However, this type of investigation requires accurate quantitative study before a final assessment of the cellular state of the cerebellar cortex can be made.

The Quantimet 720 was provided by the Association for Spina Bifida and Hydrocephalus.

\section{REFERENCES}

Balázs, R. (1972). Hormonal aspects of brain development. The Brain in Unclassified Mental Retardation, p. 61. Ed. by J. B. Cavanagh. Churchill Livingstone, Edinburgh and London.

Cole, M., and Bond, C. P. (1971). Recent advances in automatic image analysis using a television system. Fournal of Microscopy, 96, 89.

Dobbing, J., Hopewell, J. W., and Lynch, A. (1971). Vulnerability of developing brain. VII. Permanent deficit of neurons in cerebral and cerebellar cortex following early mild undernutrition. Experimental Neurology, 32, 439.

Emery, J. L., and Gadsdon, D. R. (1975). A quantitative study of the cell population of the cerebellum in children with myelomeningocele. Developmental Medicine and Child Neurology, 17, Suppl. 35, 20.

Emery, J. L., Swift, P. G. F., and Worthy, E. (1974). Hypernatraemia and uraemia in unexpected death in infancy. Archives of Disease in Childhood, 49, 686.

Gadsdon, D. R. (1976). A standardised procedure for the quantitative morphological study of the human cerebellum. Microscope (in press).

Gadsdon, D. R., and Emery, J. L. (1976a). Some quantitative morphological aspects of post-natal human cerebellar growth. fournal of Neurological Sciences, 29, 137.

Gadsdon, D. R., and Emery, J. L. (1976b). Fatty change in the brain in perinatal and unexpected death. Archives of Disease in Childhood, 51, 42.

Herndon, R. M. (1968). Thiophen induced granule cell necrosis in the rat cerebellum. Experimental Brain Research, 6, 49.

Hirono, I., Shibuya, C., and Hayashi, K. (1969). Induction of a cerebellar disorder with cyasin in newborn mice. Proceedings of the Society for Experimental Biology and Medicine, 131, 593.

Hunter, D., and Russell, D. S. (1954). Focal cerebral and cerebellar atrophy in a human subject due to organic mercury compounds. Fournal of Neurology, Neurosurgery and Psychiatry, 17, 235.

Kokenge, R., Kutt, H., and McDowell, F. (1965). Neurological

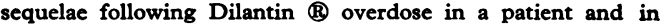
experimental animals. Neurology, 15, 823.

Krainer, L. (1949). Lamellar atrophy of Purkinje cells following heat stroke. Archives of Neurology and Psychiatry, 61, 441.

Shimada, M., and Langman, J. (1970). Repair of the external granular layer of the hamster cerebellum after prenatal and postnatal administration of methylazoxymethanol. Teratology, 3, 119.

Correspondence to Professor J. L. Emery, Children's Hospital, Western Bank, Sheffield 10. 